\title{
Impact of Repeated Application of Synthetic Insecticides on Thrips and their Natural Enemies of $B t$ Cotton
}

\author{
V. Binu* and B.V. Bhede
}

Agril. Entomology, College of Agriculture, VNMKV, Parbhani-431402, Maharashtra, India

*Corresponding author

\section{A B S T R A C T}

In order to study the effects of repeated use of various insecticides on thrips and their natural enemies viz., Ladybird beetle and Predatory spiders, an experiment was conducted during Kharif, 2018-19at the Experimental farm, Department of Agricultural Entomology,

\section{Keywords}

Cotton thrips,

Natural enemies,

Flonicamid,

Spinosad, Bt cotton

Article Info

Accepted:

04 July 2019

Available Online:

10 August 2019
Vasantrao Naik Marathwada Krishi Vidyapeeth, Parbhani. The insecticides selected included those used commonly among the farmers viz., Imidacloprid 17.8\% SL, Fipronil $5 \%$ SC, Lambda cyhalothrin 5\% EC, Spinosad 45\% SC, Acephate 75\% SP, Buprofezin $25 \%$ SC, Flonicamid 50\% WG, Acetamiprid 20\% SP, Profenofos 50\% EC, Diafenthiuron $50 \% \mathrm{WP}$, Acephate 50\% + Imidacloprid 1.8\% SP, Pyriproxyfen 5\% + Fenpropathrin 15\% EC and Profenofos $40 \%$ + Cypermethrin 4\% EC.From the first spraying onwards, Flonicamid $50 \%$ WG was found to be the best in reducing the thrips population steadily up to $0.50 /$ leaf in second spraying. But, a sudden increase in population mean was found in the third (6.80/leaf) and fourth spraying (11.50). Same trend was followed in Fipronil 5\% SC, Buprofezin 25\% SC and Diafenthiuron 50\% WP. The effect of repeated applications of synthetic insecticides on the natural enemies depicted that Spinosad $45 \% \mathrm{SC}$, Flonicamid 50\% WG and Buprofezin 25\% SC were the safest insecticides, showing maximum natural enemies population. Imidacloprid $17.8 \% \mathrm{SL}$, Fipronil $5 \% \mathrm{SC}$ and Lambda cyhalothrin $5 \%$ EC recorded minimum natural enemies population marking its intense toxicity against them.

\section{Introduction}

Cotton (Gossypium spp.) (Family: Malvaceae) popularly known as "White gold" is a major commercial crop unanimously designated as the "KING OF FIBRES" and has global significance which is grown for its lint and seed. It contains about $80 \%$ of the raw material to textile industry in the country providing livelihood for more than 100 million people, through production, processing, trading and marketing (Rakesh and Kathane, 1989). Cotton pest management has always been the most challenging task for entomologists all over the world as it suffers severe economic damage from several insects which comes around 1326 species (Matthews and Tunstall, 1994).

The decision of GEAC (Genetic Engineering and Approval Committee) of Government of India clearing the release of $B t$ cotton for commercial cultivation during 2002-2003 crop 
season, is considered as one of the major milestones in the history of cotton improvement in India. Even though this transgenic $B t$ cotton can effectively control specific lepidopterous species, there is lack of resistance against sucking pests (Hofs et al., 2004). So the sucking pests have warranted monitoring and intervention with insecticides in the early stage of the crop (Kilpatrick et al., 2005). The sucking pests including whitefly (Bemisia tabaci), Thrips (Thrips tabaci) and jassid (Amrasca biguttula biguttula) are more injurious to cotton which cause 40-50 percent damage in the crop (Naqvi, 1976). Thrips (Thrips tabaci Lind.) have already attained the status of a regular insect pest of cotton, probably due to overuse of insecticides. Thrips tabaci is the most important early season sucking insect pest of cotton. It attacks cotton crop early in the season, where both nymph and adult stages damage the tissue and destroy the crop by sucking the cell sap. This leads to the curling of leaves which eventually ends up in the stunting of plants in the initial stage. Ghabn, 1948 and Bournier, 1969 have reported that T.tabaci was responsible for the loss of $50 \%$ of young cotton plants and can also act as vector of plant diseases (Sakimura, 1963). To combat the sucking pests in India, atleast 2-3 sprays are directed against the sucking pests (Acharya et al., 2002). Farmers use higher doses on account of perception that recommended doses are not working very well in fields.

Heavy reliance and indiscriminate use of pesticides to control insect pests has led to the development of resistance to all classes of pesticides (Jayekumar and Gupta, 2000). Insecticide resistance rendered insecticides ineffectiveness necessitating repeated applications of insecticides on resorting to higher doses of insecticides, which inturn contributed to the development of resistance (Kranthi et al., 2002). Prolonged uses of the same insecticides not only elevate the problem of insecticide resistance but also disturbs the occurance of natural enemies.

\section{Materials and Methods}

The field experiment was conducted during Kharif 2018-2019, at the experimental farm of the Department of Agricultural Entomology, Vasantrao Naik Marathwada Krishi Vidyapeeth, Parbhani (Maharashtra).The repeated application of thirteen insecticides including three major combinations were selected for evaluation and comparison with untreated control. The insecticides selected included those used commonly among the farmers viz., Imidacloprid $17.8 \%$ SL, Fipronil 5\% SC, Lambda cyhalothrin 5\% EC, Spinosad 45\% SC, Acephate 75\% SP, Buprofezin 25\% SC, Flonicamid 50\% WG, Acetamiprid 20\% SP, Profenofos $50 \%$ EC, Diafenthiuron 50\%WP, Acephate 50\% + Imidacloprid 1.8\% SP, Pyriproxyfen 5\% + Fenpropathrin 15\% EC and Profenofos $40 \%+$ Cypermethrin $4 \%$ EC. The treatment details regarding the synthetic insecticides are given in the Table 1. Battery operated sprayer was used for spraying the insecticides on the crop and proper safety measures were taken while applying.

The required quantity of insecticides was mixed in water in order to prepare the spray solution. Five foliar sprays were taken at an interval of 15 days. The first spray was taken 30 days after the emergence of the crop. Care was taken to avoid the drifts to neighbouring plots. Spraying was done during the morning hours when the weather is calm and humid. The sprayer was washed after each treatment application to avoid mixing of insecticides.

The observations were recorded 1 day before spraying, then $1,3,7,14$ days after each spraying by selecting five plants randomly from each plot. The population of thrips was taken from three leaves (each from bottom, 
middle and top canopy) per plant. The natural enemy population was observed taking the whole plant into consideration.

\section{Results and Discussion}

Effect of repeated application of synthetic insecticides on Thrips, Thrips tabaci on $B t$ cotton during Kharif 2018-2019

Observations regarding the repeated application of synthetic insecticides is given in Table 2 where all treatments were found to be significantly superior over the untreated control.

Effect of different insecticides on thrips on $B t$ cotton after first spraying during Kharif 2018-2019

A uniformly distributed and low population was recorded on one day before spraying, with the population mean ranging from 2.132.93/leaf. No significant variations were seen between the treatments. The population means after the first spraying recorded Profenofos $50 \%$ EC as the best treatment in reducing thrips population $(0.02 /$ leaf). It was followed by Lambda cyhalothrin 5\% EC and Buprofezin $25 \%$ SC with a mean thrips population of 0.13 and $0.15 /$ leaf. All these were on par with Flonicamid 50\% WG, Spinosad $45 \%$ SC, Fipronil 5\% SC, Pyriproxyfen 5\% + Fenpropathrin 15\% EC, Acetamiprid 20\% SP, Profenofos $40 \%+$ Cypermethrin 4\% EC and Diafenthiuron 50\% WG.

Effect of different insecticides on thrips on $B t$ cotton after second spraying during Kharif 2018-2019

The observations after second spraying are given in the Table 2, where all treatments were found to be statistically superior over the untreated control. The mean thrips population after the second spraying depicted that Fipronil 5\% SC, Flonicamid 50\% WG, Buprofezin 25\% SC, Spinosad 45\% SC were on par with each other with mean populations of $0.42,0.50,0.55$ and $0.65 /$ leaf, respectively. The least effectiveness in reducing thrips population was noted in Pyriproxyfen 5\%+ Fenpropathrin $15 \%$ EC which was at par with Acephate $75 \%$ SP, Lambda cyhalothrin 5\% EC, Acephate 50\% + Imidacloprid 1.8\% SP and Profenofos $40 \%+$ Cypermethrin $4 \%$ EC.

Effect of different insecticides on thrips on $B t$ cotton after third spraying during Kharif 2018-2019

Flonicamid $50 \% \mathrm{WG}$ was found to be the most efficient treatment on 14 days after third spraying, marking the least thrips population. The overall mean indicated that Flonicamid $50 \%$ WG, Fipronil 5\% SC, Spinosad 45\% SC, Buprofezin 25\% SC and Diafenthiuron 50\% WP were on par and most effectrive treatments in reducing the thrips population. The next effective treatments were Acetamiprid 20\% SP, Acephate 50\% + Imidacloprid $1.8 \%$ SP, Profenofos $40 \%+$ Cypermethrin $4 \%$ EC, Lambda cyhalothrin $5 \%$ EC, Profenofos 50\% EC, Imidacloprid $17.8 \%$ SL and Pyriproxyfen 5\%+ Fenpropathrin $15 \%$ EC. The untreated control recorded significantly maximum thrips population.

\section{Effect of different insecticides on thrips on $B t$ cotton after fourth spraying during Kharif 2018-2019}

Flonicamid 50\% WG was the most effective treatment in reducing thrips population on one day after fourth spraying with a lowest mean population. The overall mean population after fourth spraying revealed that all insecticidal treatments were significantly superior over untreated control. The most effective insecticides were Flonicamid 50\% WG, 
Spinosad 45\% SC and Buprofezin 25\% SC which were on par with each other with mean populations of $11.50,15.85$ and 12.75/leaf, respectively. These were followed by Fipronil $5 \%$ SC. The least effective treatment was Acephate 50\% + Imidacloprid 1.8\% SP which was at par with Imidacloprid $17.8 \%$ SL, Lambda cyhalothrin 5\% EC and Profenofos $50 \%$ EC.

Effect of different insecticides on thrips on $B t$ cotton after fifth spraying during Kharif 2018-2019

The pooled mean data analysis after fifth spray revealed that Flonicamid 50\% WG was the most effective insecticide which marked the least population mean of $4.80 /$ leaf and was at par with Buprofezin 25\% SC (7.83/leaf) and Spinosad $45 \%$ SC (8.03/leaf). The next effective treatments were Diafenthiuron $50 \%$ WP, Pyriproxyfen 5\% + Fenpropathrin 15\% EC, Profenofos $40 \%$ + Cypermethrin $4 \%$ EC, Acephate $75 \%$ SP, Fipronil 5\% SC, Acetamiprid 20\% SP and Lambda cyhalothrin $5 \%$ EC. Acephate 50\% + Imidacloprid 1.8\% SP and Imidacloprid $17.8 \%$ SL were found to be the least efficient ones with high population means of 36.88 and 31.95/leaf, respectively among all.

\section{Effect of repeated application of different insecticides on thrips on $B t$ cotton on Kharif 2018-2019}

The effect of repeated application of different insecticides on thrips is given in the Table 2 and Figure 1 where the pretreatment count indicated no statistical difference among the treatments and the count ranged from 2.132.93/leaf. The post treatment findings indicated that all insecticidal treatments increased the thrips population gradually up to fourth spraying. Again a dip in the mean population was found during the fifth spraying. But a decline in the thrips population up to second spraying was noticed in Fipronil $5 \%$ SC, Flonicamid 50\% WG, Buprofezin $25 \% \mathrm{SC}$ and Diafenthiuron 50\% WP.

From the first spraying onwards, Flonicamid $50 \% \mathrm{WG}$ was found to be the best in reducing the thrips population steadily up to $0.50 /$ leaf in second spraying. But, a sudden increase in population mean was found in the third (6.80/leaf) and fourth spraying (11.50/leaf). Same trend was followed in Fipronil 5\% SC, Buprofezin 25\% SC and Diafenthiuron 50\% WP. The thrips population was suddenly increased after fourth and fifth spraying in Lambda cyhalothrin 5\% EC, Imidacloprid $17.8 \%$ SL and Acephate 50\%+ Imidacloprid $1.8 \% \mathrm{SP}$.

The present findings are in line with Sasikumar et al., (2015) who found Flonicamid, Diafenthiuron, Fipronil, Buprofezin to be highly effective in reducing the thrips population with higher yield. Similar results were earlier reported by Shinde and Bhede (2017) where the effect of repeated application of insecticides on thrips population showed positive results and found Flonicamid $50 \% \mathrm{WG}$ and Buprofezin 25\% SC to be the most effective in reducing population at the end of last spray. Similar findings were in agreement with Sathyan et al., (2016) who brought out the effectiveness of Fipronil 5SC and Flonicamid 50WG against T. tabaci. The data on efficacy of Diafenthiuron 50WP in reducing thrips population of cotton as reported by Bharpoda et al., (2014) also supported the above findings.

\section{Effect of application of different synthetic insecticides on Ladybird beetles on $B t$ cotton during Kharif 2018-2019}

The data in the Table 3 revealed that the population of ladybird beetle per five plants did not vary significantly at one day before spraying (pre-count) recording 0.07-0.20/plant 
indicating their uniform distribution throughout the experimental plots. On 1 day after the first spraying zero ladybird population was noticed in all treated plots. The population mean after the first spraying indicated that the Spinosad $45 \% \mathrm{SC}$ and Flonicamid 50\% WG marked the highest population of ladybird beetle $(0.55$ and $0.57 /$ plant, respectively), which was on par with the untreated control (0.53/plant). The toxicity of Lambda cyhalothrin 5\% EC was on par with Acetamiprid 20\% SP, Profenofos 50\% EC, Diafenthiuron 50\% WG, Acephate $50 \%$ + Imidacloprid 1.8\% SP, Fipronil 5\% SC, Imidacloprid 17.8\% SL and Profenofos 40\% + Cypermethrin 4\% EC.

The overall pooled data after the second spraying depicted that Flonicamid 50\% WG (0.90/plant) was the most safest insecticide for ladybird beetles, which was on par with the untreated control (1.18/plant). It was followed by Buprofezin 25\% SC (0.82/plant) and Spinosad 45\% SC (0.77/plant). Fipronil 5\% SC was observed as the most toxic treatment among all, with least population of ladybird beetle (0.07/plant), followed by Imidacloprid $17.8 \%$ SL, Acephate 50\% + Imidacloprid $17.8 \%$ SP and Lambda cyhalothrin 5\% EC.

The overall mean after the third spraying indicated that Spinosad 45\% SC and Flonicamid 50\% WG was on par with the untreated control and proved to be the safest insecticides for ladybird beetles.

There were significant differences among treatments after the fourth spraying. Spinosad $45 \%$ SC was on par with untreated control on 1 day after the fourth spraying, depicting its least toxicity towards coccinellid beetles. But, the trend was found to change a little 14 days after fourth spraying, where Spinosad 45\% EC was observed having the same population as seen in the untreated control, followed by Flonicamid 50\% EC and Buprofezin 25\% SC. The treatment with the minimum population after fourth spraying was Fipronil 5\% SC (0.02/plant), followed by Lambda cyhalothrin $5 \%$ EC (0.09/plant) and Acephate 50\% + Imidacloprid $1.8 \%$ SP (0.10/plant).

Table.1 Treatment details for field trials in $B t$ cotton

\begin{tabular}{|c|c|c|c|}
\hline Tr. No. & Treatments & $\begin{array}{c}\text { Dose } \\
\text { (g or ml/10 L) }\end{array}$ & Conc. (\%) \\
\hline $\mathbf{T}_{\mathbf{1}}$ & Imidacloprid 17.8\% SL & $4 \mathrm{ml}$ & $0.0712 \%$ \\
\hline $\mathbf{T}_{\mathbf{2}}$ & Fipronil 5\% SC & $30 \mathrm{ml}$ & $0.15 \%$ \\
\hline $\mathbf{T}_{\mathbf{3}}$ & Lambda cyhalothrin 5\%EC & $6 \mathrm{ml}$ & $0.03 \%$ \\
\hline $\mathbf{T}_{\mathbf{4}}$ & Spinosad 45\%SC & $4 \mathrm{ml}$ & $0.18 \%$ \\
\hline $\mathbf{T}_{\mathbf{5}}$ & Acephate 75 \%SP & $20 \mathrm{~g}$ & $1.5 \%$ \\
\hline $\mathbf{T}_{\mathbf{6}}$ & Buprofezin 25\% SC & $20 \mathrm{ml}$ & $0.5 \%$ \\
\hline $\mathbf{T}_{\mathbf{7}}$ & Flonicamid 50\% WG & $2 \mathrm{~g}$ & $0.1 \%$ \\
\hline $\mathbf{T}_{\mathbf{8}}$ & Acetamiprid 20\%SP & $2 \mathrm{~g}$ & $0.04 \%$ \\
\hline $\mathbf{T}_{\mathbf{9}}$ & Profenofos 50\%EC & $30 \mathrm{ml}$ & $1.5 \%$ \\
\hline $\mathbf{T}_{\mathbf{1 0}}$ & Diafenthiuron 50\%WG & $12 \mathrm{~g}$ & $0.6 \%$ \\
\hline $\mathbf{T}_{\mathbf{1 1}}$ & Acephate 50\% + Imidacloprid 1.8\%SP & $20 \mathrm{~g}$ & $1 \%+0.036 \%$ \\
\hline $\mathbf{T}_{\mathbf{1 2}}$ & Pyriproxyfen 5\%+ Fenpropathrin15\% EC & $10 \mathrm{ml}$ & $0.05 \%+0.15 \%$ \\
\hline $\mathbf{T}_{\mathbf{1 3}}$ & Profenofos 40\% + Cypermethrin 4\% EC & $20 \mathrm{ml}$ & $0.8 \%+0.08 \%$ \\
\hline $\mathbf{T}_{\mathbf{1 4}}$ & Untreated control & - & - \\
\hline
\end{tabular}


Table.2 Effect of repeated application of different insecticides on thrips on Bt cotton on Kharif 2018-2019

\begin{tabular}{|c|c|c|c|c|c|c|c|c|}
\hline \multirow[t]{2}{*}{ Tr. No } & \multirow[t]{2}{*}{ Treatments } & \multirow[t]{2}{*}{ Conc. (\%) } & \multicolumn{6}{|c|}{ Mean no. of thrips/ leaf } \\
\hline & & & $\begin{array}{l}\text { Pre- } \\
\text { count }\end{array}$ & $\begin{array}{c}\text { Spra } \\
\mathbf{y} \\
\mathbf{I}\end{array}$ & $\begin{array}{c}\text { Spray } \\
\text { II }\end{array}$ & $\begin{array}{c}\text { Spra } \\
\text { y } \\
\text { III }\end{array}$ & $\begin{array}{c}\text { Spra } \\
\text { y } \\
\text { IV }\end{array}$ & $\begin{array}{c}\text { Spra } \\
\mathbf{y} \\
\text { V }\end{array}$ \\
\hline $\mathbf{T}_{1}$ & $\begin{array}{l}\text { Imidacloprid } \\
17.8 \% \mathrm{SL}\end{array}$ & $0.0712 \%$ & $\begin{array}{c}2.33 \\
(1.82)^{*}\end{array}$ & $\begin{array}{c}0.59 \\
(1.24)\end{array}$ & $\begin{array}{c}1.37 \\
(1.50)\end{array}$ & $\begin{array}{l}14.07 \\
(3.55)\end{array}$ & $\begin{array}{l}41.00 \\
(6.28)\end{array}$ & $\begin{array}{l}31.95 \\
(5.73)\end{array}$ \\
\hline $\mathbf{T}_{2}$ & $\begin{array}{c}\text { Fipronil } \\
5 \% \mathrm{SC}\end{array}$ & $0.15 \%$ & $\begin{array}{c}2.20 \\
(1.79)\end{array}$ & $\begin{array}{c}0.30 \\
(1.13)\end{array}$ & $\begin{array}{c}0.42 \\
(1.17)\end{array}$ & $\begin{array}{c}7.53 \\
(2.66)\end{array}$ & $\begin{array}{l}24.98 \\
(4.81)\end{array}$ & $\begin{array}{l}19.63 \\
(4.47)\end{array}$ \\
\hline $\mathbf{T}_{3}$ & $\begin{array}{c}\text { Lambda cyhalothrin } \\
5 \% \mathrm{EC}\end{array}$ & $0.03 \%$ & $\begin{array}{c}2.40 \\
(1.84)\end{array}$ & $\begin{array}{c}0.13 \\
(1.06)\end{array}$ & $\begin{array}{c}2.17 \\
(1.72)\end{array}$ & $\begin{array}{l}13.30 \\
(3.47)\end{array}$ & $\begin{array}{l}39.35 \\
(5.96)\end{array}$ & $\begin{array}{l}26.73 \\
(5.22)\end{array}$ \\
\hline $\mathrm{T}_{4}$ & $\begin{array}{c}\text { Spinosad } \\
\mathbf{4 5} \% \mathrm{SC}\end{array}$ & $0.18 \%$ & $\begin{array}{c}2.13 \\
(1.77)\end{array}$ & $\begin{array}{c}0.28 \\
(1.12)\end{array}$ & $\begin{array}{c}0.65 \\
(1.27)\end{array}$ & $\begin{array}{c}7.95 \\
(2.73)\end{array}$ & $\begin{array}{l}12.75 \\
(3.65)\end{array}$ & $\begin{array}{l}8.03 \\
(2.94)\end{array}$ \\
\hline $\mathrm{T}_{5}$ & $\begin{array}{l}\text { Acephate } \\
75 \% \text { SP }\end{array}$ & $1.5 \%$ & $\begin{array}{c}2.93 \\
(1.98)\end{array}$ & $\begin{array}{c}0.44 \\
(1.18)\end{array}$ & $\begin{array}{c}2.37 \\
(1.77)\end{array}$ & $\begin{array}{l}12.72 \\
(3.51)\end{array}$ & $\begin{array}{l}29.60 \\
(5.33)\end{array}$ & $\begin{array}{l}19.25 \\
(4.47)\end{array}$ \\
\hline $\mathrm{T}_{6}$ & $\begin{array}{c}\text { Buprofezin } \\
25 \% \text { SC }\end{array}$ & $0.5 \%$ & $\begin{array}{c}2.20 \\
(1.79)\end{array}$ & $\begin{array}{c}0.15 \\
(1.07)\end{array}$ & $\begin{array}{c}0.55 \\
(1.23)\end{array}$ & $\begin{array}{c}8.78 \\
(2.83)\end{array}$ & $\begin{array}{l}15.85 \\
(4.04)\end{array}$ & $\begin{array}{c}7.33 \\
(2.82)\end{array}$ \\
\hline $\mathbf{T}_{7}$ & $\begin{array}{c}\text { Flonicamid } \\
50 \% \text { WG }\end{array}$ & $0.1 \%$ & $\begin{array}{c}2.53 \\
(1.88)\end{array}$ & $\begin{array}{c}0.18 \\
(1.08)\end{array}$ & $\begin{array}{c}0.50 \\
(1.21)\end{array}$ & $\begin{array}{c}6.80 \\
(2.59)\end{array}$ & $\begin{array}{l}11.50 \\
(3.49)\end{array}$ & $\begin{array}{c}4.80 \\
(2.36)\end{array}$ \\
\hline $\mathrm{T}_{\mathrm{Q}}$ & $\begin{array}{c}\text { Acetamiprid } \\
20 \% \mathrm{SP}\end{array}$ & $0.04 \%$ & $\begin{array}{c}2.40 \\
(1.84)\end{array}$ & $\begin{array}{c}0.32 \\
(1.14)\end{array}$ & $\begin{array}{c}0.83 \\
(1.33)\end{array}$ & $\begin{array}{l}11.52 \\
(3.18)\end{array}$ & $\begin{array}{l}30.67 \\
(5.50)\end{array}$ & $\begin{array}{l}20.68 \\
(4.59)\end{array}$ \\
\hline $\mathrm{T}_{9}$ & $\begin{array}{c}\text { Profenofos } \\
50 \% \text { EC }\end{array}$ & $1.5 \%$ & $\begin{array}{c}2.60 \\
(1.89)\end{array}$ & $\begin{array}{c}0.02 \\
(1.01)\end{array}$ & $\begin{array}{c}1.09 \\
(1.42)\end{array}$ & $\begin{array}{l}13.48 \\
(3.46)\end{array}$ & $\begin{array}{l}32.38 \\
(5.57)\end{array}$ & $\begin{array}{l}18.35 \\
(4.32)\end{array}$ \\
\hline $\mathrm{T}_{10}$ & $\begin{array}{c}\text { Diafenthiuron } \\
50 \% \text { WP }\end{array}$ & $0.6 \%$ & $\begin{array}{c}2.67 \\
(1.91)\end{array}$ & $\begin{array}{c}0.29 \\
(1.13)\end{array}$ & $\begin{array}{c}0.77 \\
(1.31)\end{array}$ & $\begin{array}{c}9.37 \\
(2.94)\end{array}$ & $\begin{array}{l}25.12 \\
(5.00)\end{array}$ & $\begin{array}{l}13.93 \\
(3.77)\end{array}$ \\
\hline $\mathbf{T}_{11}$ & $\begin{array}{c}\text { Acephate } 50 \%+ \\
\text { Imidacloprid } 1.8 \\
\text { \%SP }\end{array}$ & $\begin{array}{c}1 \%+ \\
0.036 \%\end{array}$ & $\begin{array}{c}2.93 \\
(1.98)\end{array}$ & $\begin{array}{c}0.37 \\
(1.16)\end{array}$ & $\begin{array}{c}1.97 \\
(1.67)\end{array}$ & $\begin{array}{l}12.83 \\
(3.44)\end{array}$ & $\begin{array}{l}43.20 \\
(6.45)\end{array}$ & $\begin{array}{l}36.88 \\
(6.13)\end{array}$ \\
\hline $\mathrm{T}_{12}$ & $\begin{array}{c}\text { Pyriproxyfen 5\%+ } \\
\text { Fenpropathrin } 15 \% \mathrm{E} \\
\text { C }\end{array}$ & $\begin{array}{r}0.05 \% \\
+0.15 \%\end{array}$ & $\begin{array}{c}2.13 \\
(1.77)\end{array}$ & $\begin{array}{c}0.28 \\
(1.13)\end{array}$ & $\begin{array}{c}2.38 \\
(1.78)\end{array}$ & $\begin{array}{l}15.75 \\
(3.74)\end{array}$ & $\begin{array}{l}29.17 \\
(5.34)\end{array}$ & $\begin{array}{l}14.70 \\
(3.91)\end{array}$ \\
\hline $\mathbf{T}_{13}$ & $\begin{array}{c}\text { Profenofos } 40 \%+ \\
\text { Cypermethrin } 4 \% \\
\text { EC }\end{array}$ & $\begin{array}{l}0.8 \%+ \\
0.08 \%\end{array}$ & $\begin{array}{c}2.53 \\
(1.88)\end{array}$ & $\begin{array}{c}0.32 \\
(1.14)\end{array}$ & $\begin{array}{l}1.79 \\
(1.62)\end{array}$ & $\begin{array}{l}13.07 \\
(3.44)\end{array}$ & $\begin{array}{l}30.03 \\
(5.36)\end{array}$ & $\begin{array}{l}16.92 \\
(4.17)\end{array}$ \\
\hline $\mathbf{T}_{14}$ & Untreated control & & $\begin{array}{c}2.60 \\
(1.90)\end{array}$ & $\begin{array}{l}1.10 \\
(1.41)\end{array}$ & $\begin{array}{l}3.58 \\
(2.10)\end{array}$ & $\begin{array}{l}18.62 \\
(4.43)\end{array}$ & $\begin{array}{l}75.30 \\
(8.43)\end{array}$ & $\begin{array}{l}76.27 \\
(8.67)\end{array}$ \\
\hline \multicolumn{3}{|c|}{$\mathrm{SE} \pm$} & 0.10 & 0.05 & 0.08 & 0.13 & 0.32 & 0.24 \\
\hline \multicolumn{3}{|c|}{ CD@ 5\% } & NS & 0.15 & 0.24 & 0.39 & 0.91 & 0.69 \\
\hline \multicolumn{3}{|c|}{$\mathbf{C V}$} & 9.41 & 9.48 & 10.96 & 8.26 & 11.83 & 10.61 \\
\hline
\end{tabular}

*Figures in parenthesis are $\sqrt{(X+1)}$ transformed values 
Table.3 Effect of repeated application of different insecticides on ladybird beetles on $B t$ cotton on Kharif 2018-2019

\begin{tabular}{|c|c|c|c|c|c|c|c|c|}
\hline \multirow[t]{2}{*}{ Tr. No } & \multirow[t]{2}{*}{ Treatments } & \multirow[t]{2}{*}{ Conc.(\%) } & \multicolumn{6}{|c|}{ Mean no. of ladybird beetles/plant } \\
\hline & & & $\begin{array}{l}\text { Pre- } \\
\text { count }\end{array}$ & $\begin{array}{c}\text { Spra } \\
\text { y I }\end{array}$ & $\begin{array}{c}\text { Spra } \\
\mathbf{y} \\
\text { II }\end{array}$ & $\begin{array}{c}\text { Spra } \\
\mathbf{y} \\
\text { III }\end{array}$ & $\begin{array}{l}\text { Spray } \\
\text { IV }\end{array}$ & $\begin{array}{c}\text { Spra } \\
\mathbf{y} \\
\mathbf{V}\end{array}$ \\
\hline $\mathbf{T}_{1}$ & $\begin{array}{l}\text { Imidacloprid } \\
17.8 \% \text { SL }\end{array}$ & $0.0712 \%$ & $\begin{array}{c}0.20 \\
(1.09) \\
*\end{array}$ & $\begin{array}{c}0.25 \\
(1.11)\end{array}$ & $\begin{array}{c}0.15 \\
(1.07)\end{array}$ & $\begin{array}{l}0.08 \\
(1.04)\end{array}$ & $\begin{array}{c}0.12 \\
(1.05)\end{array}$ & $\begin{array}{c}0.07 \\
(1.03)\end{array}$ \\
\hline $\mathbf{T}_{2}$ & $\begin{array}{l}\text { Fipronil } \\
\text { 5\% SC }\end{array}$ & $0.15 \%$ & $\begin{array}{c}0.20 \\
(1.09)\end{array}$ & $\begin{array}{c}0.22 \\
(1.10)\end{array}$ & $\begin{array}{c}0.07 \\
(1.03)\end{array}$ & $\begin{array}{c}0.05 \\
(1.02)\end{array}$ & $\begin{array}{c}0.02 \\
(1.00)\end{array}$ & $\begin{array}{c}0.03 \\
(1.01)\end{array}$ \\
\hline $\mathbf{T}_{3}$ & $\begin{array}{l}\text { Lambda cyhalothrin } \\
5 \% \text { EC }\end{array}$ & $0.03 \%$ & $\begin{array}{c}0.13 \\
(1.06)\end{array}$ & $\begin{array}{c}0.10 \\
(1.05)\end{array}$ & $\begin{array}{c}0.18 \\
(1.08)\end{array}$ & $\begin{array}{c}0.15 \\
(1.07)\end{array}$ & $\begin{array}{c}0.09 \\
(1.04)\end{array}$ & $\begin{array}{c}0.12 \\
(1.05)\end{array}$ \\
\hline $\mathrm{T}_{4}$ & $\begin{array}{l}\text { Spinosad } \\
45 \% \mathrm{SC}\end{array}$ & $0.18 \%$ & $\begin{array}{c}0.20 \\
(1.08)\end{array}$ & $\begin{array}{c}0.55 \\
(1.23)\end{array}$ & $\begin{array}{c}0.77 \\
(1.32)\end{array}$ & $\begin{array}{c}1.00 \\
(1.41)\end{array}$ & $\begin{array}{c}0.62 \\
(1.26)\end{array}$ & $\begin{array}{c}0.17 \\
(1.07)\end{array}$ \\
\hline $\mathbf{T}_{5}$ & $\begin{array}{l}\text { Acephate } \\
75 \% \text { SP }\end{array}$ & $1.5 \%$ & $\begin{array}{c}0.13 \\
(1.06)\end{array}$ & $\begin{array}{c}0.40 \\
(1.18)\end{array}$ & $\begin{array}{c}0.30 \\
(1.13)\end{array}$ & $\begin{array}{c}0.37 \\
(1.16)\end{array}$ & $\begin{array}{c}0.18 \\
(1.08)\end{array}$ & $\begin{array}{c}0.07 \\
(1.03)\end{array}$ \\
\hline $\mathrm{T}_{6}$ & $\begin{array}{l}\text { Buprofezin } \\
25 \% \mathrm{SC}\end{array}$ & $0.5 \%$ & $\begin{array}{c}0.20 \\
(1.08)\end{array}$ & $\begin{array}{c}0.39 \\
(1.16)\end{array}$ & $\begin{array}{c}0.82 \\
(1.34)\end{array}$ & $\begin{array}{c}0.68 \\
(1.29)\end{array}$ & $\begin{array}{c}0.43 \\
(1.19)\end{array}$ & $\begin{array}{c}0.12 \\
(1.05)\end{array}$ \\
\hline $\mathbf{T}_{7}$ & $\begin{array}{l}\text { Flonicamid } \\
\mathbf{5 0 \%} \text { WG }\end{array}$ & $0.1 \%$ & $\begin{array}{c}0.20 \\
(1.08)\end{array}$ & $\begin{array}{c}0.57 \\
(1.23)\end{array}$ & $\begin{array}{c}0.90 \\
(1.37)\end{array}$ & $\begin{array}{c}0.80 \\
(1.34)\end{array}$ & $\begin{array}{c}0.57 \\
(1.25)\end{array}$ & $\begin{array}{c}0.17 \\
(1.07)\end{array}$ \\
\hline $\mathrm{T}_{8}$ & $\begin{array}{l}\text { Acetamiprid } \\
20 \% \text { SP }\end{array}$ & $0.04 \%$ & $\begin{array}{c}0.13 \\
(1.06)\end{array}$ & $\begin{array}{c}0.20 \\
(1.09)\end{array}$ & $\begin{array}{c}0.20 \\
(1.09)\end{array}$ & $\begin{array}{c}0.22 \\
(1.10)\end{array}$ & $\begin{array}{c}0.17 \\
(1.07)\end{array}$ & $\begin{array}{l}0.08 \\
(1.04)\end{array}$ \\
\hline$T_{9}$ & $\begin{array}{l}\text { Profenofos } \\
50 \% \text { EC }\end{array}$ & $1.5 \%$ & $\begin{array}{c}0.20 \\
(1.09)\end{array}$ & $\begin{array}{c}0.20 \\
(1.09)\end{array}$ & $\begin{array}{c}0.27 \\
(1.12)\end{array}$ & $\begin{array}{c}0.20 \\
(1.09)\end{array}$ & $\begin{array}{c}0.10 \\
(1.05)\end{array}$ & $\begin{array}{l}0.08 \\
(1.04)\end{array}$ \\
\hline $\mathrm{T}_{10}$ & $\begin{array}{l}\text { Diafenthiuron } \\
50 \% \text { WP }\end{array}$ & $0.6 \%$ & $\begin{array}{c}0.07 \\
(1.03)\end{array}$ & $\begin{array}{c}0.22 \\
(1.09)\end{array}$ & $\begin{array}{c}0.50 \\
(1.21)\end{array}$ & $\begin{array}{c}0.53 \\
(1.23)\end{array}$ & $\begin{array}{c}0.20 \\
(1.09)\end{array}$ & $\begin{array}{c}0.12 \\
(1.05)\end{array}$ \\
\hline $\mathrm{T}_{11}$ & $\begin{array}{c}\text { Acephate 50\% + } \\
\text { Imidacloprid } 1.8 \% \text { SP }\end{array}$ & $\begin{array}{c}1 \%+ \\
0.036 \%\end{array}$ & $\begin{array}{c}0.13 \\
(1.06)\end{array}$ & $\begin{array}{c}0.20 \\
(1.09)\end{array}$ & $\begin{array}{c}0.15 \\
(1.07)\end{array}$ & $\begin{array}{c}0.25 \\
(1.11)\end{array}$ & $\begin{array}{c}0.10 \\
(1.04)\end{array}$ & $\begin{array}{l}0.08 \\
(1.04)\end{array}$ \\
\hline $\mathrm{T}_{12}$ & $\begin{array}{c}\text { Pyriproxyfen 5\%+ } \\
\text { Fenpropathrin15\% EC }\end{array}$ & $\begin{array}{c}0.05 \%+0 \\
15 \%\end{array}$ & $\begin{array}{c}0.20 \\
(1.09)\end{array}$ & $\begin{array}{c}0.38 \\
(1.17)\end{array}$ & $\begin{array}{c}0.48 \\
(1.21)\end{array}$ & $\begin{array}{c}0.40 \\
(1.18)\end{array}$ & $\begin{array}{c}0.17 \\
(1.08)\end{array}$ & $\begin{array}{c}0.10 \\
(1.04)\end{array}$ \\
\hline $\mathrm{T}_{13}$ & $\begin{array}{l}\text { Profenofos } 40 \%+ \\
\text { Cypermethrin } 4 \% \text { EC }\end{array}$ & $\begin{array}{l}0.8 \%+ \\
0.08 \%\end{array}$ & $\begin{array}{c}0.20 \\
(1.08)\end{array}$ & $\begin{array}{c}0.30 \\
(1.13)\end{array}$ & $\begin{array}{c}0.27 \\
(1.12)\end{array}$ & $\begin{array}{c}0.25 \\
(1.11)\end{array}$ & $\begin{array}{c}0.15 \\
(1.07)\end{array}$ & $\begin{array}{c}0.07 \\
(1.03)\end{array}$ \\
\hline $\mathrm{T}_{14}$ & Untreated control & & $\begin{array}{c}0.13 \\
(1.06)\end{array}$ & $\begin{array}{c}0.53 \\
(1.22)\end{array}$ & $\begin{array}{c}1.18 \\
(1.47)\end{array}$ & $\begin{array}{l}1.05 \\
(1.42)\end{array}$ & $\begin{array}{c}0.73 \\
(1.31)\end{array}$ & $\begin{array}{c}0.25 \\
(1.11)\end{array}$ \\
\hline \multicolumn{3}{|c|}{ SE \pm} & 0.07 & 0.03 & 0.02 & 0.03 & 0.02 & 0.01 \\
\hline \multirow{2}{*}{\multicolumn{3}{|c|}{ CD@ 5\% }} & NS & 0.09 & 0.07 & 0.08 & 0.07 & NS \\
\hline & & & 10.67 & 6.04 & 4.05 & 4.77 & 4.30 & 2.01 \\
\hline
\end{tabular}

*Figures in parenthesis are $\sqrt{(X+1)}$ transformed values 
Table.4 Effect of repeated application of different insecticides on predatory spiders on $B t$ cotton during Kharif 2018-2019

\begin{tabular}{|c|c|c|c|c|c|c|c|c|}
\hline \multirow{2}{*}{$\begin{array}{l}\text { Tr. } \\
\text { No }\end{array}$} & \multirow[t]{2}{*}{ Treatments } & \multirow[t]{2}{*}{ Conc. $(\%)$} & \multicolumn{6}{|c|}{ Mean No. of predatory spiders/ plant } \\
\hline & & & $\begin{array}{l}\text { Pre- } \\
\text { count }\end{array}$ & $\begin{array}{c}\text { Spra } \\
\text { y I }\end{array}$ & $\begin{array}{c}\text { Spra } \\
\mathbf{y} \\
\mathbf{I I}\end{array}$ & $\begin{array}{c}\text { Spra } \\
\mathbf{y} \\
\text { III }\end{array}$ & $\begin{array}{c}\text { Spra } \\
\mathbf{y} \\
\text { IV }\end{array}$ & $\begin{array}{c}\text { Spray } \\
\text { V }\end{array}$ \\
\hline $\mathbf{T}_{1}$ & $\begin{array}{c}\text { Imidacloprid } \\
17.8 \% \text { SL }\end{array}$ & $0.0712 \%$ & $\begin{array}{c}0.00 \\
(1.00)^{*}\end{array}$ & $\begin{array}{c}0.07 \\
(1.03)\end{array}$ & $\begin{array}{c}0.13 \\
(1.06)\end{array}$ & $\begin{array}{c}0.40 \\
(1.18)\end{array}$ & $\begin{array}{c}0.52 \\
(1.23)\end{array}$ & $\begin{array}{c}0.52 \\
(1.23)\end{array}$ \\
\hline $\mathbf{T}_{2}$ & $\begin{array}{c}\text { Fipronil } \\
\mathbf{5 \%} \text { SC }\end{array}$ & $0.15 \%$ & $\begin{array}{c}0.00 \\
(1.00)\end{array}$ & $\begin{array}{c}0.07 \\
(1.03)\end{array}$ & $\begin{array}{c}0.15 \\
(1.07)\end{array}$ & $\begin{array}{c}0.27 \\
(1.12)\end{array}$ & $\begin{array}{c}0.28 \\
(1.13)\end{array}$ & $\begin{array}{c}0.24 \\
(1.11)\end{array}$ \\
\hline$T_{3}$ & $\begin{array}{c}\text { Lambda cyhalothrin } \\
5 \% \text { EC }\end{array}$ & $0.03 \%$ & $\begin{array}{c}0.00 \\
(1.00)\end{array}$ & $\begin{array}{c}0.05 \\
(1.03)\end{array}$ & $\begin{array}{c}0.05 \\
(1.02)\end{array}$ & $\begin{array}{c}0.25 \\
(1.11)\end{array}$ & $\begin{array}{c}0.30 \\
(1.14)\end{array}$ & $\begin{array}{c}0.22 \\
(1.10)\end{array}$ \\
\hline $\mathrm{T}_{4}$ & $\begin{array}{l}\text { Spinosad } \\
45 \% \mathrm{SC}\end{array}$ & $0.18 \%$ & $\begin{array}{c}0.00 \\
(1.00)\end{array}$ & $\begin{array}{c}0.28 \\
(1.12)\end{array}$ & $\begin{array}{c}0.44 \\
(1.19)\end{array}$ & $\begin{array}{c}1.28 \\
(1.50)\end{array}$ & $\begin{array}{c}1.40 \\
(1.55)\end{array}$ & $\begin{array}{c}1.22 \\
(1.49)\end{array}$ \\
\hline$T_{5}$ & $\begin{array}{l}\text { Acephate } \\
75 \% \mathrm{SP}\end{array}$ & $1.5 \%$ & $\begin{array}{c}0.00 \\
(1.00)\end{array}$ & $\begin{array}{c}0.13 \\
(1.06)\end{array}$ & $\begin{array}{c}0.37 \\
(1.16)\end{array}$ & $\begin{array}{c}0.78 \\
(1.33)\end{array}$ & $\begin{array}{c}0.68 \\
(1.29)\end{array}$ & $\begin{array}{c}0.67 \\
(1.28)\end{array}$ \\
\hline $\mathrm{T}_{6}$ & $\begin{array}{l}\text { Buprofezin } \\
\mathbf{2 5 \%} \mathrm{SC}\end{array}$ & $0.5 \%$ & $\begin{array}{c}0.00 \\
(1.00)\end{array}$ & $\begin{array}{c}0.23 \\
(1.10)\end{array}$ & $\begin{array}{c}0.58 \\
(1.24)\end{array}$ & $\begin{array}{c}1.32 \\
(1.51)\end{array}$ & $\begin{array}{c}1.24 \\
(1.49)\end{array}$ & $\begin{array}{c}1.12 \\
(1.45)\end{array}$ \\
\hline $\mathbf{T}_{7}$ & $\begin{array}{c}\text { Flonicamid } \\
50 \% \text { WG }\end{array}$ & $0.1 \%$ & $\begin{array}{c}0.00 \\
(1.00)\end{array}$ & $\begin{array}{c}0.21 \\
(1.09)\end{array}$ & $\begin{array}{c}0.55 \\
(1.23)\end{array}$ & $\begin{array}{c}1.28 \\
(1.51)\end{array}$ & $\begin{array}{c}1.27 \\
(1.50)\end{array}$ & $\begin{array}{c}1.18 \\
(1.47)\end{array}$ \\
\hline $\mathrm{T}_{8}$ & $\begin{array}{l}\text { Acetamiprid } \\
20 \% \mathrm{SP}\end{array}$ & $0.04 \%$ & $\begin{array}{c}0.00 \\
(1.00)\end{array}$ & $\begin{array}{c}0.11 \\
(1.05)\end{array}$ & $\begin{array}{c}0.17 \\
(1.08)\end{array}$ & $\begin{array}{c}0.67 \\
(1.28)\end{array}$ & $\begin{array}{c}0.54 \\
(1.23)\end{array}$ & $\begin{array}{c}0.58 \\
(1.26)\end{array}$ \\
\hline$T_{9}$ & $\begin{array}{l}\text { Profenofos } \\
50 \% \text { EC }\end{array}$ & $1.5 \%$ & $\begin{array}{c}0.00 \\
(1.00)\end{array}$ & $\begin{array}{c}0.07 \\
(1.03)\end{array}$ & $\begin{array}{c}0.12 \\
(1.05)\end{array}$ & $\begin{array}{c}0.32 \\
(1.14)\end{array}$ & $\begin{array}{c}0.23 \\
(1.10)\end{array}$ & $\begin{array}{c}0.30 \\
(1.14)\end{array}$ \\
\hline $\mathrm{T}_{10}$ & $\begin{array}{c}\text { Diafenthiuron } \\
50 \% \text { WP }\end{array}$ & $0.6 \%$ & $\begin{array}{c}0.00 \\
(1.00)\end{array}$ & $\begin{array}{c}0.15 \\
(1.07)\end{array}$ & $\begin{array}{c}0.47 \\
(1.21)\end{array}$ & $\begin{array}{c}0.96 \\
(1.39)\end{array}$ & $\begin{array}{c}0.97 \\
(1.40)\end{array}$ & $\begin{array}{c}0.85 \\
(1.36)\end{array}$ \\
\hline $\mathrm{T}_{11}$ & $\begin{array}{c}\text { Acephate 50\% + } \\
\text { Imidacloprid } 1.8 \% \mathrm{SP}\end{array}$ & $1 \%+0.036 \%$ & $\begin{array}{c}0.00 \\
(1.00)\end{array}$ & $\begin{array}{c}0.08 \\
(1.04)\end{array}$ & $\begin{array}{c}0.17 \\
(1.07)\end{array}$ & $\begin{array}{c}0.47 \\
(1.20)\end{array}$ & $\begin{array}{c}0.63 \\
(1.27)\end{array}$ & $\begin{array}{c}0.63 \\
(1.28)\end{array}$ \\
\hline $\mathrm{T}_{12}$ & $\begin{array}{c}\text { Pyriproxyfen 5\%+ } \\
\text { Fenpropathrin15\%EC }\end{array}$ & $0.05 \%+0.15 \%$ & $\begin{array}{c}0.00 \\
(1.00)\end{array}$ & $\begin{array}{c}0.13 \\
(1.06)\end{array}$ & $\begin{array}{c}0.28 \\
(1.13)\end{array}$ & $\begin{array}{c}0.72 \\
(1.30)\end{array}$ & $\begin{array}{c}0.82 \\
(1.34)\end{array}$ & $\begin{array}{c}0.82 \\
(1.35)\end{array}$ \\
\hline$T_{13}$ & $\begin{array}{c}\text { Profenofos } 40 \%+ \\
\text { Cypermethrin 4\% EC }\end{array}$ & $0.8 \%+0.08 \%$ & $\begin{array}{c}0.00 \\
(1.00)\end{array}$ & $\begin{array}{c}0.19 \\
(1.08)\end{array}$ & $\begin{array}{c}0.08 \\
(1.04)\end{array}$ & $\begin{array}{c}0.35 \\
(1.15)\end{array}$ & $\begin{array}{c}0.43 \\
(1.19)\end{array}$ & $\begin{array}{c}0.43 \\
(1.19)\end{array}$ \\
\hline $\mathrm{T}_{14}$ & Untreated control & & $\begin{array}{c}0.00 \\
(1.00)\end{array}$ & $\begin{array}{c}0.31 \\
(1.13)\end{array}$ & $\begin{array}{c}0.88 \\
(1.36)\end{array}$ & $\begin{array}{c}1.58 \\
(1.60)\end{array}$ & $\begin{array}{c}1.52 \\
(1.59)\end{array}$ & $\begin{array}{c}1.37 \\
(1.54)\end{array}$ \\
\hline \multicolumn{3}{|c|}{$\mathbf{S E} \pm$} & 0.00 & 0.03 & 0.03 & 0.03 & 0.03 & 0.03 \\
\hline \multicolumn{3}{|c|}{ CD@ 5\% } & 0.00 & 0.07 & 0.10 & 0.08 & 0.10 & 0.09 \\
\hline \multicolumn{3}{|c|}{ CV } & 0.00 & 5.23 & 6.45 & 4.47 & 5.00 & 4.54 \\
\hline
\end{tabular}

*Figures in parenthesis are $\sqrt{ }(\mathrm{X}+1)$ transformed values 
Fig.1 Effect of repeated application of different insecticides on thrips on Bt cotton during Kharif 2018-2019

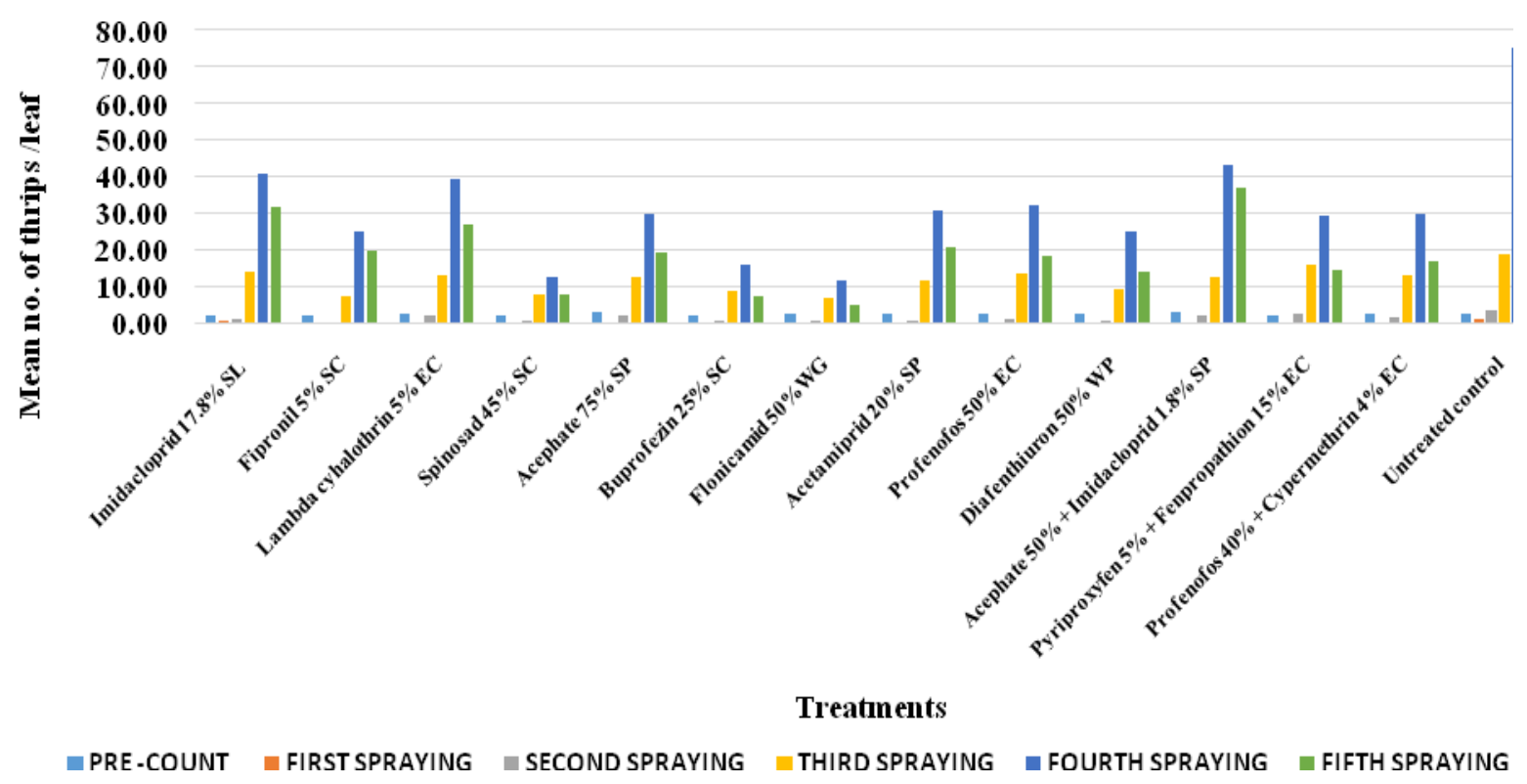

Fig.2 Effect of repeated application of different insecticides on Ladybird beetles on $B t$ cotton during Kharif 2018-2019

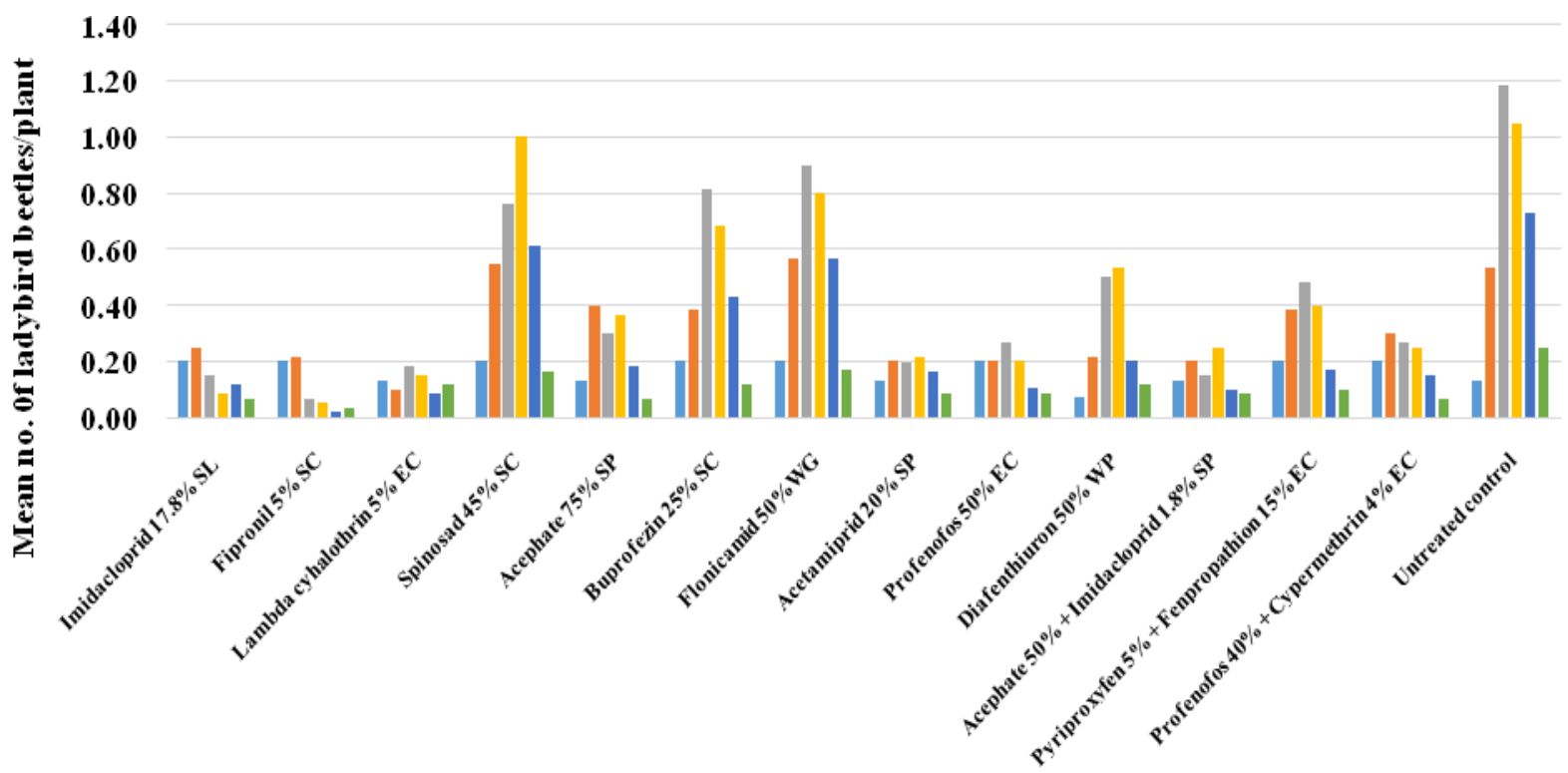

Treatments

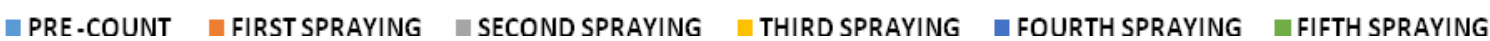


Fig.3 Effect of repeated application of different insecticides on predatory spiders on $B t$ cotton during Kharif 2018-2019

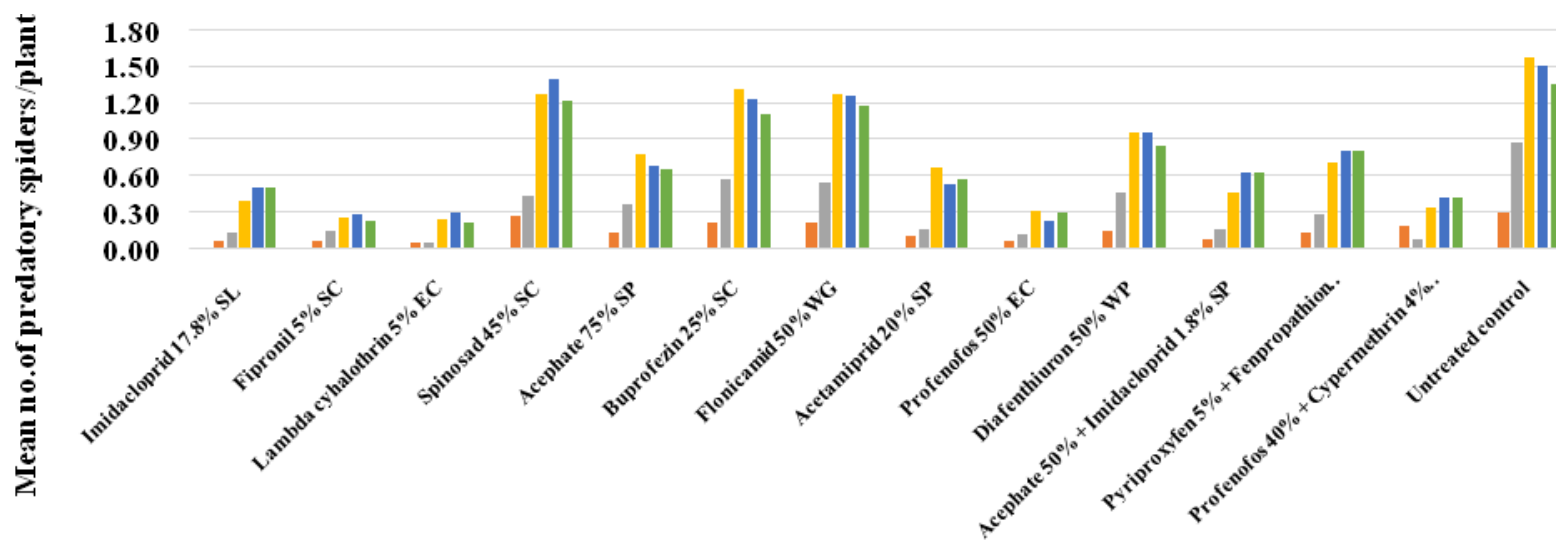

Treatments

\#PRE-COUNT $\square$ FIRST SPRAYING $\square$ SECOND SPRAYING

"THIRD SPRAYING $\square$ FOURTH SPRAYING $\square$ FIFTH SPRAYING

The population was very high after fifth spraying. Spinosad $45 \%$ SC marked same population mean as that of Flonicamid $50 \%$ WG (0.17/plant) and was on par with Buprofezin 25\% SC (0.12/plant). The most toxic treatment with least population was Imidacloprid $17.8 \%$ SL (0.07/plant) which was equally efficient with Fipronil 5\% SC, Acetamiprid $20 \%$ SP, Pyriproxyfen $5 \%+$ Fenpropathrin 15\% EC and Profenofos $40 \%+$ Cypermethrin 4\% EC.

Effect of repeated spraying of different insecticides on ladybird beetles on Btcotton during Kharif 2018-19.

The pooled analysis of all sprayings on ladybird beetles are presented in the Table 3 and Figure 2, where no significant difference in the population was observed 1 day before the first spraying. But, after the first spray, some of the treatments were on par with the untreated control.

The overall recordings emphasized that the effect of Imidacloprid $17.8 \%$ SL was toxic and reduced the ladybird population after each sprayings. Its effect was seen on par with Fipronil 5\% SC and Lambda cyhalothrin 5\% EC. The population was observed to steadily keep in pace with the untreated control in case of Flonicamid $50 \%$ WG.

The present findings are in tune with Nemade et al., (2017) who reported that Flonicamid is the promising insecticide for aphid control in term of selectivity for aphid antagonist, the ladybird beetle. The findings of Chandi et al., (2016) also supports this observations, where they reported the mortality of coccinellids on the sucking pests of $B t$ cotton to be significantly less in Flonicamid 50\% WG and more in Imidacloprid $17.8 \%$ SL.

Effect of application of different synthetic insecticides on Predatory spiders on $B t$ cotton during Kharif 2018-2019

The data in the Table 4 revealed that no predatory spiders were seen 1 day before spraying and 1 and 3 days after the first spraying. The overall mean after the first spraying revealed that Spinosad $45 \%$ SC was the least toxic treatment to the predatory 
spiders (0.28/plant) which were on par with Buprofezin 25\% SC (0.23/plant), Flonicamid $50 \%$ WG (0.21/plant), Profenofos $40 \%+$ Cypermethrin 4\% EC, Acephate $75 \%$ SP and Diafenthiuron $50 \%$ WG. Lambda cyahalothrin 5\% EC marked the lowest population mean of $0.05 /$ leaf.

The pooled data after the second spraying indicated that Spinosad $45 \%$ SC was the safest insecticide for predatory spiders (0.44/plant), whose effect on the predatory spiders was on par with the untreated control (0.88/plant). The most toxic treatment was Lambda cyhalothrin 5\% EC, marking lowest predatory spider population of $0.05 /$ plant, followed by Profenofos $40 \%+$ Cypermethrin $4 \%$ EC (0.08/plant).

An increase in the population was observed after third spraying when compared to first spraying. The overall mean after the third spraying depicted that Flonicamid 50\% WG (1.28/plant) and Buprofezin 25\% SC (1.32/plant) was safer to the predatory spiders and were on par with the untreated control (1.58/plant). Lambda cyhalothrin 5\% EC was observed as the most toxic for predatory spiders followed by Fipronil 5\% SC.

The pooled data on fourth spraying reported that Spinosad 45\% SC, Flonicamid 50\% WG and Buprofezin 25\% SC were safer to spiders. Profenofos $50 \%$ EC was observed as the most toxic towards predatory spiders, followed by Fipronil 5\% SC and Lambda cyhalothrin 5\% EC.

The pooled data on fifth spraying recorded maximum population of predatory spiders. The overall data on the fifth spraying indicated that Spinosad 45\% SC (1.22/plant), Flonicamid $50 \%$ WG (1.18/plant) and Buprofezin 25\% SC (1.12/plant) were safer and on par with the untreated control (1.37/plant). Lambda cyhalothrin 5\% EC was recorded as the most toxic insecticide for predatory spiders, followed by Fipronil 5\% SC and Profenofos 50\% EC.

Effect of repeated spraying of different insecticides on Predatory spiders on $B t$ cotton during Kharif2018-19.

The effect of repeated application of different insecticides on predatory spiders is given in the Table 4 and Figure 3. No predatory spiders were seen on 1 day before first spraying, the population increased gradually after each sprayings. The population increase was very slow in Lambda cyhalothrin 5\% EC and Profenofos 50\% EC up to second spraying. From third spraying onwards, a steady population increase was observed. Maximum population was observed in Spinosad $45 \%$ SC, followed by Flonicamid $50 \% \mathrm{WG}$ and Buprofezin 25\% SC. The effect of Spinosad $45 \%$ SC was no different from the untreated control. The present findings are in tune with Murray and Lloyd (1997), who reported that there were no differences in the predator spider populations between unsprayed and Spinosad treated plots and a substantial reduction in the population of predatory spiders was seen in the conventional treatments.

\section{Thrips, Thripstabaci}

From the first spraying onwards, Flonicamid $50 \% \mathrm{WG}$ was found to be the best in reducing the thrips population steadily up to second spraying. But, a sudden increase in population mean was found in the third and fourth spraying. Same trend was followed in Fipronil $5 \%$ SC, Buprofezin $25 \% \quad \mathrm{SC}$ and Diafenthiuron $50 \%$ WP. Flonicamid, Diafenthiuron, Fipronil, Buprofezin were highly effective in reducing the thrips population when compared to all other treatments 


\section{Ladybird beetles}

The overall recordings emphasizes the toxic effect of Imidacloprid $17.8 \%$ SL in reducing the Ladybird population after each sprayings. Its effect was seen on par with Fipronil 5\% SC and Lambda cyhalothrin 5\% EC. The population was observed to steadily keep in pace with the untreated control in case of Flonicamid 50\% WG and Spinosad 45\% SC.

\section{Predatory spiders}

Maximum population was observed in Spinosad 45\% SC, followed by Flonicamid $50 \% \mathrm{WG}$ and Buprofezin 25\% SC. The effect of Spinosad $45 \%$ SC was no different from the untreated control.

The effect of repeated applications of synthetic insecticides on the natural enemies depicted that Spinosad 45\% SC, Flonicamid $50 \% \mathrm{WG}$ and Buprofezin 25\% SC were the safest insecticides, showing maximum natural enemies population. Imidacloprid $17.8 \% \mathrm{SL}$, Fipronil 5\% SC and Lambda cyhalothrin 5\% EC recorded minimum natural enemies population marking its intense toxicity against them.

\section{References}

Acharya, S., Mishra, H.P and Dash, D. (2002). Efficacy of insecticides against okra jassid, Amrasca biguttula biguttula. J. of Cotton Res. Dev., 10(2): 230-232.

Bharpoda, T.M., Patel, N.B., Thumar, R.K., Bhatt; Ghetiya, L.V., Patel, H.C and Board, P.K. (2014). Evaluation of insecticides against sucking pests infesting $B t$ Cotton Boll Guard II. The Bioscan - An International Quarterly Journal of Life Sciences, 9(3): 977-980.

Bournier, A. and Couilloud, R. (1969). Les Thrips du cotonnieren Iran. Cotton et
Fibres Tropicals, 24: 211-8.

Chandi, R.S., Kumar, V., Bhullar, H.S and Dhawan, A.K. (2016). Field efficacy of Flonicamid 50WG against sucking insect pests and predatory complex on Bt cotton. Ind. J. of Pl. Prot., 44(1): 1-8.

Ghabn, A.A. (1948). Contribution to the knowledge of biology of T.tabaci in Egypt. Bulletin de La Societe Fouad ler of Entomologie, 32: 123-74

Hofs, J.L; Schoeman, A and Vaissayre, M. (2004). Effect of Bt cotton on arthropod biodiversity in south African cotton yield. Common Agril. Appl. Biol. Sci., 69: 191-194.

Jeyakumar, P and Gupta, G.P. (2000). Utilization of Neem and $B t$ for managing bollworms in cottons. Ind. J. of Ento., 64: 424-33.

Kilpatrick, A.L., Hagerty, S.G., Turnipseed, Sullivan, M.J and W.C, Jr. Bridges (2005). Activity of selected neonicotinoids and dicrotophos on nontarget arthropods in cotton: implications in insect management. J. Econ. Entomol., 98(3): 814-820.

Kranthi K.R., Jadhav, D.R., Wanjari, R.R., Ali; Shakir, Russell, D. (2002). Insecticide resistance in five major insect pests of cotton in India. Crop Prot., 21: 449-60.

Matthews, G.A and Tunstall, J.P. (Eds) (1994). Insect pests of cotton. CAB International, Wallingford, UK. 593 pp.

Murray, D.A.H and Lloyd, R.J. (1997). The effect of Spinosad (Tracer) on arthropod pests and beneficial population in Australian cotton. Proceedings, Beltwide cotton Conferences, New Orleans, L.A, USA, Jan 6-10, vol 2: 1087-1091.

Naqvi, K.M. (1976). Crop protection to boost up the cotton production. Seminar organized by ESSO, Fert. Co. Ltd. Pakistan

Nemade, P.W., Rathod, T.H., Deshmukh, 
S.B., Ujjainkar, V.V and Deshmukh, V.V. (2017). Evaluation of new molecules against sucking pests of $B t$ cotton. J. of Ent. and Zoo. Studies, 5(6): 659-663.

Rakesh, R.C and Kathane, T.V. (1989). Cotton marketing federation and export of cotton in India (1980-1981 to 19871988). Cott. Dev., 18(3-4): 1-18.

Sakimura, K., (1963). Frankliniella fusca, an additional vector for tomato spotted wilt virus, with notes on Thrips tabaci, another vector. Phytopathol., 53: 412415.

Sasikumar, K., Gunasekaran, M and Shanthi,
M. (2015). Bioefficacy of insecticides against sucking pests in $B t$-cotton. J. of Cotton Res. and Dev., 32(2): 269-275.

Sathyan, T., Murugesan, N., Elanchezhyan, K., Arockia S.R.J., Ravi, G. (2016). Efficacy of synthetic insecticides against sucking pests in cotton, Gossypium hirsutum L. Int.J. of Ento. Res., 1(1): 16-21.

Shinde, V.D. and Bhede, B.V. (2018). Effect of indiscriminate use of insecticides on status of pests and their natural enemies in $B t$ cotton. M.Sc. Thesis (2017). Vasantrao Naik Marathwada University, Parbhani.

\section{How to cite this article:}

Binu, V. and Bhede, B.V. 2019. Impact of Repeated Application of Synthetic Insecticides on Thrips and their Natural Enemies of Bt Cotton. Int.J.Curr.Microbiol.App.Sci. 8(08): 277-289. doi: https://doi.org/10.20546/ijcmas.2019.808.033 